\title{
Identification of genes regulated by glucose in a pancreatic beta-cell line by a new method for subtraction of mRNA
}

\author{
E. Yamato ${ }^{1}$, H. Ikegami ${ }^{1}$, J.-I. Miyazaki ${ }^{2}$, T. Ogihara ${ }^{1}$ \\ ${ }^{1}$ Department of Geriatric Medicine, Osaka University Medical School, Osaka, Japan \\ ${ }^{2}$ Department of Disease-Related Gene Regulation Research (Sandoz), Faculty of Medicine, University of Tokyo, Tokyo, Japan
}

\begin{abstract}
Summary Various genes are differentially expressed in cells during cell differentiation, development, aging, and in pathological conditions. To identify and isolate the genes that are specifically and differentially expressed in cells, we established a ligation-mediated polymerase chain reaction (PCR)-based method for cDNA subtraction. As this method is PCRbased, when target genes are expressed at high levels relative to the driver (a control pool for subtraction), even a small amount of target genes can be amplified. By this newly developed PCR-based subtraction
\end{abstract}

method, a set of genes regulated by glucose were identified in a mouse insulinoma cell line. This PCRbased and non-radioactive subtraction method will be a powerful tool for identification of novel genes, specifically and differentally expressed in cells. [Diabetologia (1996) 39: 1293-1298]

Keywords Glucose-regulated gene, cDNA subtraction, gene expression, polymerase chain reaction, expressed sequence tag.
Mammalian cells possess about 100000 different genes of which approximately 15000 are expressed in each cell. Inducible and tissue-specific gene expression in an individual cell is required for its development and differentiation and is essential for an appropriate response to stimuli in order to adapt to the environment [1]. As inappropriate expression of genes is known to cause disease, it is important to investigate changes in gene expression.

Non-insulin-dependent diabetes mellitus is a heterogeneous and multi-factorial disorder and one of the major characteristics of the disease is an

Received: 20 March 1996 and in final revised form: 22 July 1996

Corresponding author: E. Yamato, M.D., Ph.D., Department of Geriatric Medicine, Osaka University Medical School, 2-2 Yamadaoka, Suita, Osaka 565, Japan

Abbreviations: PCR, Polymerase chain reaction; RT, reverse transcriptase; RDA, representational difference analysis; HGIS, high glucose inducible sequence; LGIS, low glucose inducible sequence; IAPP, islet amyloid polypeptide; TTR, transthyretin; UBQOXR, ubiquinone oxidoreductase; EST, expressed sequence tags. impairment of glucose-induced insulin secretion from pancreatic beta cells. Since inappropriate expression of a set of genes might correlate with impaired responsiveness of pancreatic beta cells to glucose, identification of the genes regulated by glucose will lead to further understanding of the pathogenesis and pathophysiology of the disease.

To identify and isolate the specific genes that are differentially expressed in individual cells, the subtraction hybridization method has been widely used for cDNA subtraction [2-4]. However, this method is complicated, takes a long time to screen thousands of clones and needs a large amount of mRNA. The differential display method of mRNA has recently been developed and several new genes have been identified [5]. This method is useful, but it requires isotopes and sequence gel to isolate cDNA.

To simplify subtraction cloning, we have established the ligation-mediated polymerase chain reaction (PCR)-based method for cDNA subtraction, in which a small population of mRNA can be identified by a PCR-mediated technique without the use of an isotope. The representational difference analysis (RDA) method has been developed for identification 
of restriction endonuclease fragments present in one population of DNA fragments of the genome, but not in another [6]. We adapted the strategies of RDA designed for genomic DNA analysis to cDNA subtraction. In this study, we applied the newly developed PCR-based cDNA subtraction method to the identification of genes regulated by glucose and successfully identified a group of genes regulated by glucose from a mouse pancreatic beta-cell line.

\section{Materials and methods}

Culture of MIN6 cells. MIN6 cells were maintained in Dulbecco's modified Eagle's medium containing $25 \mathrm{mmol} / \mathrm{l}$ glucose and $13 \%$ heat-inactivated fetal bovine serum in humidified $5 \% \mathrm{CO}_{2} / 95 \% \mathrm{O}_{2}$ at $37^{\circ} \mathrm{C}$ [7]. Cells at the 17 th passage were used for the experiment.

Subtraction procedure. The outline of the ligation-mediated PCR-based method for cDNA subtraction method is described in Figure 1. Driver (which is a control pool for subtraction) and tracer (which includes the cDNA of interest) cDNA libraries were prepared from mRNAs of cell or tissue sources and each group of cDNAs was ligated to adapter-primers, which have a cleavage site of a certain restriction endonuclease, at both blunted ends of cDNAs. PCR was performed on the cDNAs of each library, resulting in amplification of size-restricted cDNAs (shorter than several kilobases in size). Following digestion of each pool of cDNAs with the restriction endonuclease, only fragments of tracer cDNA were ligated to new adapter-primer set at both their $5^{\prime}$ ends. Then a small amount of tracer is mixed with an excess of driver, and the mixture is denatured and reannealed. Most of the tracers, which were expressed at the same level as the driver, such as housekeeping genes, were hybridized with an excess amount of complementary driver, whereas some populations of target cDNAs, which were expressed at high levels in a tracer, show self-reannealing. Only self-reannealed tracer cDNAs, which have adapter-primer at both ends, can be amplified by PCR. Subtracted-amplified cDNAs were then digested with restriction endonuclease to remove the adapter-primers and ligated to a new set of adapter-primers. An exponential enrichment of target cDNAs was obtained by several rounds of subtraction and amplification steps.

Subtraction-amplification procedure by the ligation-mediated PCR-based method for cDNA subtraction was performed with cDNAs from mRNAs derived from MIN6 cells. MIN6 cells were cultured in high $(25 \mathrm{mmol} / \mathrm{l})$ or low $(2.5 \mathrm{mmol} / \mathrm{l}) \mathrm{glu}-$ cose medium for $24 \mathrm{~h}$ and RNAs were extracted by the guanidine thiocyanate-phenol method. Double-stranded cDNAs were prepared by oligo dT primed method from mRNA purified with an oligo dT column. Following ligation to BamHI/ NotI linker (5'-GAT CCG CGG CCG C-3', Pharmacia, Uppsala, Sweden), $1 \mu \mathrm{g}$ of dsDNAs was mixed with $0.5 \mathrm{nmol}$ of primer-adapters (R12 and R24, R series) in $30 \mu \mathrm{l}$ of T4 DNA ligase buffer (Wako Nippon Gene, Tokyo, Japan). Oligonucleotides were annealed by cooling gradually for $50^{\circ} \mathrm{C}$ to $10^{\circ} \mathrm{C}$ for $1 \mathrm{~h}$ and ligated by incubation with $500 \mathrm{U}$ of T4 DNA ligase (Wako Nippon Gene) overnight. Primer-adapters used in this experiment have been reported by Lisitsyn and colleagues [6] (R24: 5'-AGC ACT CTC CAG CCC TCA CCG AG-3', R12: 5'-GAT CCT CGG TGA-3'). Complementary DNAs were amplified with R24 primer for 20 cycles (80 ng of template DNA with $300 \mu \mathrm{mol} / \mathrm{l}$ of each dNTP, $0.01 \%$ bovine serum

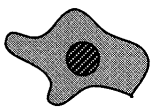

RNA extraction

cDNA synthesis

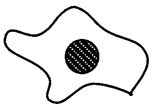

RNA extraction cDNA synthesis

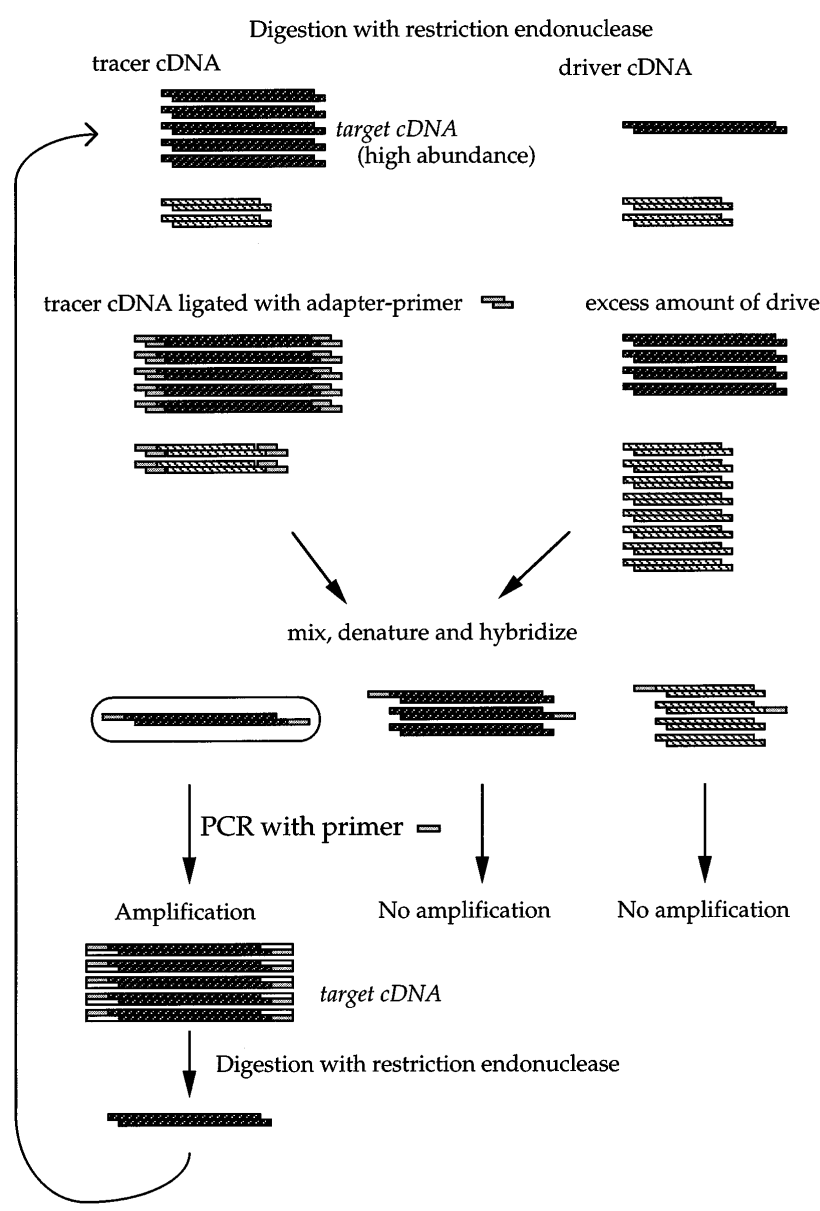

Fig. 1. Schema of the ligation-mediated PCR-based method for cDNA subtraction

albumin, $67 \mathrm{mmol} / \mathrm{l}$ Tris- $\mathrm{HCl}, \mathrm{pH} \quad 8.8,4 \mathrm{mmol} / \mathrm{l} \quad \mathrm{MgCl}_{2}$ $16 \mathrm{mmol} / \mathrm{l}\left(\mathrm{NH}_{4}\right)_{2} \mathrm{SO}_{4}, 10 \mathrm{mmol} / \mathrm{l} \beta$-mercaptoethanol, $1 \mu \mathrm{mol} / \mathrm{l}$ of forward and reverse primers in $400 \mu \mathrm{l} \mathrm{PCR}$ reaction, $1 \mathrm{~min}$ at $95^{\circ} \mathrm{C}, 2 \mathrm{~min}$ at $52^{\circ} \mathrm{C}, 7 \mathrm{~min}$ at $72^{\circ} \mathrm{C}$ ) in a thermal cycler (Perkin-Elmer Cetus, Foster City, Calif., USA). The amplified cDNAs were purified with Microcon 100 (Amicon, Beverly, Mass., USA) and digested with BamHI. After removal of adapter-primers by Microcon 100, digested cDNA of interest was ligated to new adapter-primers (J24: 5'-ACC GAC GTC GAC TAT CCA TGA ACG-3', J12; 5'-GAT CCG TTC ATG-3', J series) as above, while control cDNAs were left without adapter-primers. Then $1 \mu \mathrm{g}$ of cDNA of interest with adapter-primer and $20 \mu \mathrm{g}$ of cDNA of control without primeradapters were mixed and precipitated with ethanol. Precipitate was disolved in $4 \mu \mathrm{l}$ of $3 \times$ EE buffer [6] and hybridized at $67^{\circ} \mathrm{C}$ overnight. Hybridized DNA was amplified with J24 primer for 10 cycles $\left(1 \mathrm{~min}\right.$ at $95^{\circ} \mathrm{C}, 3 \mathrm{~min}$ at $72^{\circ} \mathrm{C}, \mathrm{PCR}$ condition was described as above). After incubation with mung bean nuclease to degrade the single-stranded DNA, subtracted cDNA of interest was amplified with J24 primer for 20 cycles ( 1 min at $95^{\circ} \mathrm{C}, 3 \mathrm{~min}$ at $70^{\circ} \mathrm{C}$, PCR condition was described as above). Following digestion with BamHI, amplified subtracted cDNA was applied to Microcon 100 to remove the adapter-primers. 
Digested subtracted cDNA was ligated to other adapter-primers (N24: 5 '-AGG CAA CTG TGC TAT CCG AGG GAG, N12: 5 '-GAT CCT CCC TCG-3', N series), then the second subtraction procedure was performed as for the first subtraction step. The third subtraction step with $\mathrm{J}$ series primers, fourth step with $\mathrm{N}$ series, fifth step with $\mathrm{J}$ series, sixth step with $\mathrm{N}$ series, and seventh step with $\mathrm{J}$ series were performed. The adapters for PCR were altered between rounds of subtraction and amplification to avoid the accumulation of PCR products that might interfere with subsequent amplification [6].

Sequence analysis of subtracted clones. Subtracted PCR products were electrophoreted by agarose gel and hybridized with rat preproinsulin I gene. Then the subtracted PCR products excluding those of insulin cDNA by size were recovered by diethylaminoethyl (DEAE) paper. Following exclusion of cDNA fragments, recovered cDNAs were digested with BamHI. Subtracted cDNAs were subcloned into pUC119. Colonies were picked up from subtracted cDNA libraries and inserted cDNA was amplified with primers, both ends of pUC119 of multi-cloning site (M13 reverse primer: 5'-GTT TTC CCA GTC ACG AC-3' and M13 primer: 5'-CAG GAA ACA GCT ATG AC-3') for 25 cycles $\left(30 \mathrm{~s}\right.$ at $94^{\circ} \mathrm{C}, 2 \mathrm{~min}$ at $55^{\circ} \mathrm{C}$, $1 \mathrm{~min}$ at $72^{\circ} \mathrm{C}$ ). Following removal of primers with Microcon 100 , amplified inserted cDNAs were sequenced by 373A sequencer (Applied Biosystems, Foster City, Calif., USA) with cycle-sequencing protocol using PCR with dye primers (M13 or M13 reverse). The sequence of subtracted cDNA was compared to those in European Molecular Biology Laboratory (EMBL) and GenBank data libraries with basic local alignment search tool (BLAST) programs of the National Center for Biotechnology Information (NCBI).

RT-PCR of UBQOXR and CAN genes. Total RNA was extracted from adult $\mathrm{C} 57 \mathrm{BL} / 6$ mouse tissues by the guanidine thiocyanate-phenol method; $10 \mu \mathrm{g}$ was then reverse-transcribed and amplified by a thermal cycler for 40 cycles. Primers used for the PCR are described below. UBQOXR $(30 \mathrm{~s}$ at $95^{\circ} \mathrm{C}, 1 \mathrm{~min}$ at $65^{\circ} \mathrm{C}, 2 \mathrm{~min}$ at $72^{\circ} \mathrm{C}$ ): sense primer, $5^{\prime}$-CGC TGG TGG TGT CCT TCT CT- $3^{\prime}$ and antisense primer, $5^{\prime}$ TGA GGG TGG CTG GGC ACA TC- $3^{\prime}$. CAN (30 $\mathrm{s}$ at $95^{\circ} \mathrm{C}, 1 \mathrm{~min}$ at $60^{\circ} \mathrm{C}, 2 \mathrm{~min}$ at $72^{\circ} \mathrm{C}$ ): sense primer, $5^{\prime}$-CCC AAA TAC CTC TAA TCT GTC T- $3^{\prime}$ and antisense primer, 5'-ACA CTG GAG CAG CAC CGA A-3'. Amplified RTPCR product was subjected to $9 \%$ polyacrylamide gel electrophoresis and stained with ethidium bromide.

\section{Results}

Subtraction procedures. Subtraction-amplification was performed with cDNAs from mRNAs derived from MIN6 cells, established as a pancreatic betacell line, which retains glucose-induced insulin secretion [7]. Since glucose may alter expression of a variety of genes in MIN6 cells, we applied the RDA method to MIN-6 cells, cultured under high and low glucose conditions to identify the glucoseinducible mRNAs and glucose-static mRNAs. A detectable increase of subtracted cDNAs in the fragment size distribution was observed by ethidium bromide staining (Fig. 2). Subtracted cDNA libraries were established from a mixture of cDNAs from the 6 th and 7 th subtraction-amplification steps. Inserted
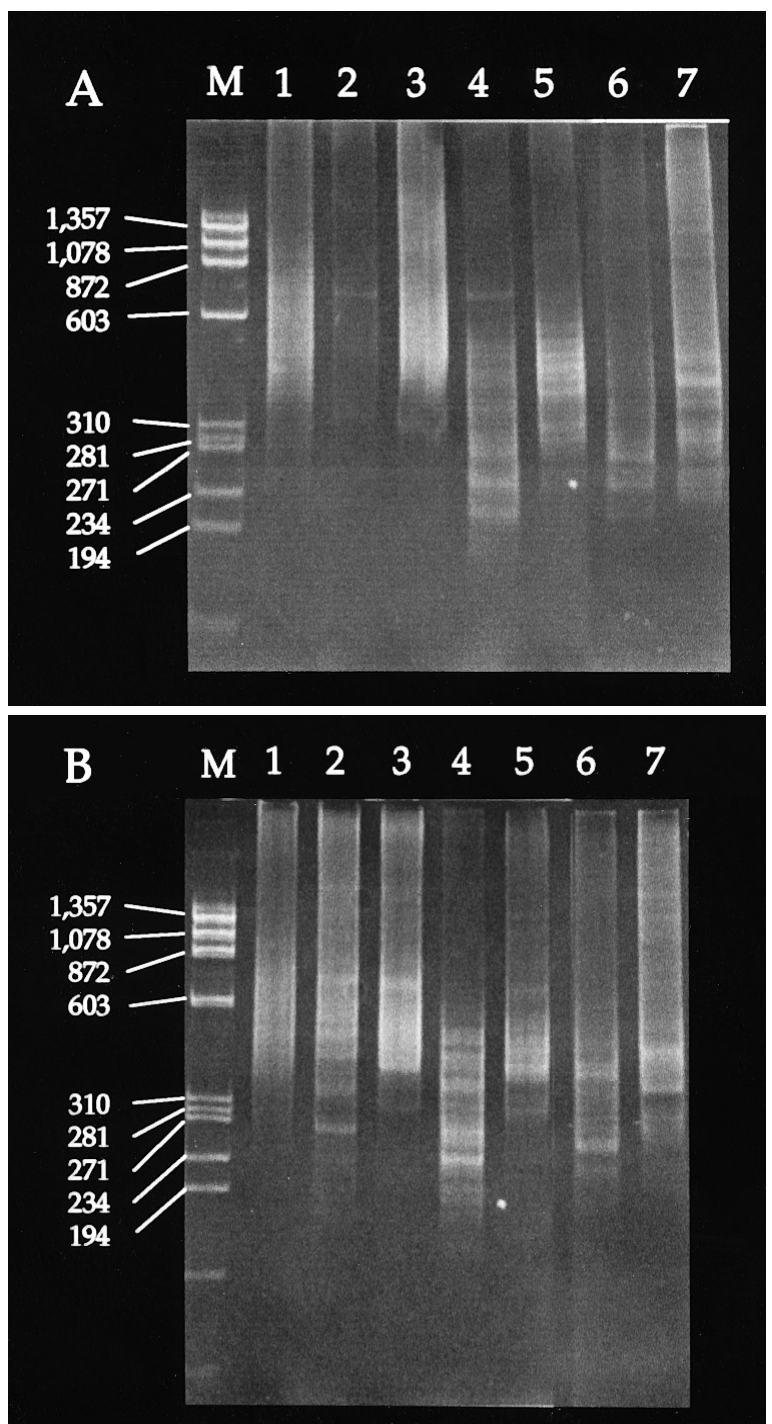

Fig. 2.A, B. Representational subtraction analysis with mRNAs from MIN6 cells. The subtracted cDNAs, which were preferentially expressed in MIN6 cells cultured in (A) high glucose medium $(25 \mathrm{mmol} / \mathrm{l}$ for $24 \mathrm{~h})$ and in (B) low glucose medium $(2.5 \mathrm{mmol} / \mathrm{l}$ for $24 \mathrm{~h})$, obtained by sequential subtraction steps were subjected to $9 \%$ polyacrylamide gel electrophoresis and stained with ethidium bromide. M Size-marker, HaeIII digest of ${ }_{\varnothing} \mathrm{X} 174 \mathrm{RF}$ DNA. Lanes 1-7: first to seventh subtraction-amplification steps of subtraction. DNAs with odd numbers $(1,3,5,7)$ were amplified with $\mathrm{J}$ series primer and DNAs with even numbers $(2,4,6)$ were amplified with $\mathrm{N}$ series primer. Sizes (base pairs) are indicated on the left

cDNAs were amplified by PCR and subjected to sequencing.

Analysis of subtracted clones. Out of 19 clones screened from the cDNA library, representing preferentially expressed mRNAs in the MIN6 cells cultured in high glucose medium, we identified 16 individual cDNA clones (high glucose inducible sequence, HGIS) and out of 11 clones screened from the cDNA library, representing preferentially expressed 


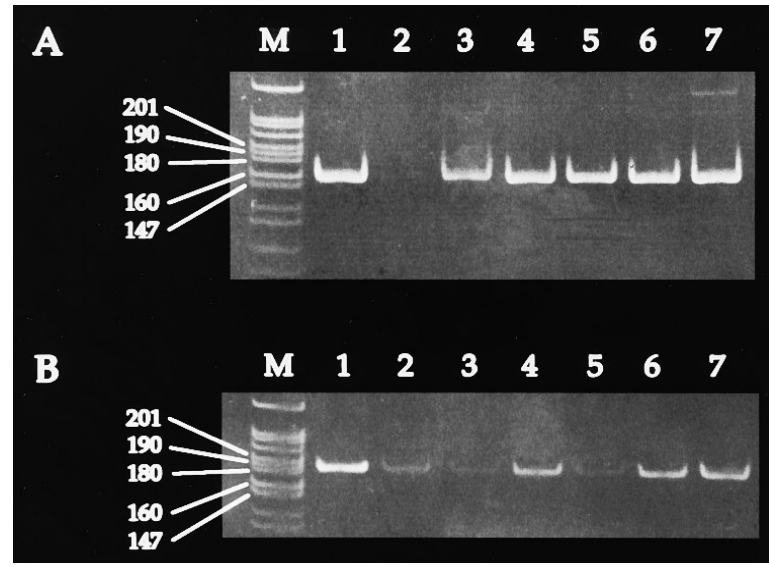

Fig.3.A, B. RT-PCR analysis of UBQOXR-B9 (A) and CAN (B) mRNA in MIN6 cells and mouse tissues: MIN6 cells cultured in high (1) and low glucose (2), brain (3), pancreas (4), kidney (5), liver (6), and spleen (7), respectively. M: size-marker, HaeIII digest of ${ }_{\varnothing}$ X174 RF DNA. Sizes (base pairs) are indicated on the left. Amplified DNA was subjected to $9 \%$ polyacrylamide gel electrophoresis and stained with ethidium bromide

Table 1. Summary of identified genes and their frequencies in the subtracted clones

HGIS library: 19 clones

Known genes

2 mouse IAPP

1 mouse TTR

Unknown genes

homologous to genes of other species

2 human EST48844 T32463

2 H. sapiens partial cDNA F08678

1 bovine ubiquinone oxidoreductase chain CI-B9

1 H. sapiens CAN mRNA

no homology to other genes

10

LGIS library: 11 clones

Known genes

3 mouse intracisternal-A particle

2 mouse EST GM2A12

2 mouse XPB/ERCC3

Unknown genes

homologous to genes of other species

2 H. sapiens cDNA clone F12165

1 H.sapiens cDNA clone T84551

no homology to other genes

1

mRNAs in the MIN6 cells cultured in low glucose medium, we identified 6 individual cDNA clones (low glucose inducible sequence, LGIS) (Table 1). In HGIS, 10 individual sequences out of 16 clones were found to be novel genes $(62.5 \%)$ and two clones were identical to the sequences in the database, mouse islet amyloid polypeptide (IAPP) cDNA [8] and mouse transthyretin (TTR) cDNA [9]. Northern analysis confirmed the increase in expression of IAPP and TTR gene in MIN6 cells under high glucose conditions when compared to those under low glucose conditions, indicating that our subtraction method had been successfully performed. Four clones were identified as being homologous sequences to other species, two of which were homologous to human expressed sequence tag and the other two clones were homologous to known genes of other species. Clone HGIS29 was homologous to bovine ubiquinone oxidoreductase (UBQOXR) subunit B9 [10] $(78 \%)$ and clone HGIS 20 was homologous to Homo sapiens CAN mRNA [11] (85\%).

In LGIS, each two clones were found to be identical to mouse XPB/ERCC3 gene [12] and mouse expressed sequence tag GM2A12. Three clones had a $90 \%$ homologous sequence to a family of defective murine retrovirus-like elements known as intracisternal A particles [13]. The sequences of two clones were homologous to human expressed sequence tag and one clone was found to be a novel gene (Table 1).

RT-PCR of UBQOXR and CAN gene homologues. To confirm the expression of UBQOXR and CAN gene homologues in normal tissues, reverse transcriptasePCR (RT-PCR) was performed with RNAs from various mouse tissues as well as MIN6 cells cultured under high or low glucose conditions (Fig. 3). The UBQOXR and CAN genes were expressed with relatively high abundance in MIN6 cells cultured with high glucose, whereas only subtle expression was found in MIN6 cells cultured with low glucose, when compared to the expression of $\beta$-actin gene as an internal standard. In addition, same samples were subjected to Northern blot analysis with $\beta$-actin probe to check that the amount of total RNA for templates of RT-PCR was properly controlled. Since both the UBQOXR and CAN genes were identified in HGIS, this result indicates that the subtraction procedure was successfully performed.

A single band with expected size of RT-PCR products of both the UBQOXR and the CAN genes was obtained from RNAs of several mouse tissues and MIN6 cells. The UBQOXR gene was expressed in all the tissues examined, brain, pancreas, liver, spleen, and kidney with similar abundance. On the other hand the expression of the CAN gene showed tissuespecific heterogeneity. The CAN gene was expressed with relatively high abundance in mouse pancreas, liver and kidney, but with low abundance in mouse brain and liver.

\section{Discussion}

We have developed a new ligation-mediated PCRbased method for cDNA subtraction. The advantage of this subtraction method is not only its simplicity, but also its effectiveness to amplify even target cDNAs with low abundance when the target is 
expressed at a high level relative to the driver. Since this method is a PCR-based technique, the absolute amount of target cDNA is no longer a limiting factor. Only a relative abundance of target to driver is required for detection and isolation of the target. Recently the usefulness of our strategy was also confirmed by Hubank and Schatz [14], who successfully isolated the caffeine up-regulated clones from the pre-beta-cell lines.

Using the newly developed PCR-based method for cDNA subtraction, we have identified 16 individual clones in HGIS and 6 in LGIS. There was no overlap in sequences between HGIS and LGIS subtracted cDNA pools.

In HGIS, two clones had identical sequences in the database, mouse IAPP cDNA [8] and mouse TTR cDNA [9]. Previous studies revealed that IAPP is localized in secretory granules in the pancreatic beta cells and that IAPP mRNA level is increased in islets cultured under high glucose conditions [15]. This previous finding confirmed that cDNA clones that were preferentially expressed in MIN6 cells under high glucose conditions were successfully subtracted by our method. The TTR gene encodes prealbumin and in situ analysis revealed that TTR mRNA is localized in pancreatic islets [16-18]. Although the function of TTR in the islets is unknown, our data suggest that the expression of TTR is controlled by glucose.

Two individual clones homologous to genes in other species, bovine UBQOXR B9 subunit and human CAN genes, were identified in HGIS. UBQOXR is a part of NADH-Q oxidoreductase (also known as complex I). NADH-Q oxidoreductase is the first enzyme in the respiratory electron transport chain of mitochondria and the B9 subunit of bovine UBQOXR is a nuclear coded subunit [10]. RT-PCR analysis confirmed that UBQOXR gene expression was augmented by glucose. As UBQOXR is crucial for glucose-dependent ATP synthesis in the TCA cycle, and glucose-induced insulin secretion in pancreatic beta cells is accompanied by an increase of ATP, UBQOXR gene expression may have a role in glucoseinduced insulin secretion.

The CAN gene was identified from a human cDNA library as a gene related to a chromosomal translocation associated with leukaemia $[11,19]$. A structure of $\beta$ turns at the C-terminal part of the CAN protein may function as an ancillary DNA binding domain [11]. Although the function of CAN has not been fully elucidated, domains identified in these proteins suggested a role in regulation of transcription. Our results indicated that the CAN gene was expressed in pancreatic beta cells and its expression was increased by glucose. These data suggest that the CAN gene product may contribute to the glucose-induced gene regulation in pancreatic beta cells.

In our study, several clones homologous to the genes previously assigned as expressed sequence tags (ESTs) were identified: two individual clones in HGIS homologous to human ESTs, and two individual clones homologous to human ESTs and one to mouse EST in LGIS. Large-scale cDNA sequencing analysis in various tissues has been performed to examine the organ-specific EST as a part of the human and mouse genome projects [20-25]. Our method revealed that parts of the genes previously assigned as ESTs were homologous to the sequences of subtracted clones. This result implies that those ESTs were expressed in pancreatic beta cells and regulated by glucose although further analysis for expression of these subtracted genes should be needed in more physiological conditions, such as isolated beta cells or isolated islets. Therefore, our strategy will be useful for further characterization of the ESTs whose function and/or function regulation is unknown.

Besides four sequences homologous to known ESTs, we have identified 10 novel sequences in HGIS and 1 in LGIS in MIN6 cells. Recently, the random cDNA sequencing approach has been performed on RNAs from human islet [26] and MIN6 cells [27]. Although a large number of novel genes were obtained in those studies, the newly identified sequences identified in this paper did not show any homology with those ESTs. Moreover, our method can eliminate uninformative housekeeping genes through the subtraction procedure. The differential display method amplifies the fragment of all represented cDNAs, whereas our method can excluded those cDNAs and amplify only the differences. Therefore our approach, in addition to the random cDNA cloning method, will greatly increase the substantial resources of cDNA and aid in the development of a physical map and isolation of candidate disease genes. Further investigation will be needed to determine the physiological role of the novel genes in insulin secretion from pancreatic beta cells and to examine whether these genes could be candidate genes conferring susceptibility to non-insulin-dependent diabetes.

As our subtraction method is PCR-based, even a subtle amount of target can be amplified when the target is expressed at a high level relative to the driver. Moreover, as our subtraction method needs no isotope for subtraction, the experimental procedure is simple and easy. Therefore, this method is expected to be a useful tool for identification of novel genes, specifically and differentially expressed in cell differentiation, aging, cancer, cardiovascular disease and other common diseases.

Acknowledgements. This work was supported in part by a Grant for Diabetes Research from the Ministry of Health and Welfare, a Grant-in-Aid of the Japan Medical Association, a grant from Otsuka Pharmaceutical Co., a grant from the Mitsubishi Grant-in-Aid and a grant from Sandoz Foundation for Gerontological Research. 
The nucleotide sequence data reported in this paper will appear in the DDBJ, EMBL, and GenBank nucleotide sequence databases with the following accession numbers; D84271, D84175, D84176, D84177, D84178, D84179, D84180, D84181, D84182, D84183, D84184, D84185, D84186.

\section{References}

1. Maniatis T, Goodbourn S, Fischer JA (1987) Regulation of inducible and tissue-specific gene expression. Science 236: 1237-1245

2. Timblin C, Battey J, Kuehl WM (1990) Application for PCR technology to subtractive cDNA cloning: identification of genes expressed specifically in murine plasmacytoma cells. Nucleic Acids Res 18: 1587-1593

3. Sargent TD, Dawid IB (1983) Differential gene expression in the gastrula of Xenopus laevis. Science 222: 135-139

4. Schneider C, King RM, Philipson L (1988) Genes specifically expressed at growth arrest of mammalian cells. Cell 54: 787-793

5. Liang P, Pardee AB (1992) Differential display of eukaryotic messenger RNA by means of the polymerase chain reaction. Science 257: 967-971

6. Lisitsyn N, Lisitsyn N, Wigler M (1993) Cloning the differences between two complex genomes. Science 259: 946951

7. Miyazaki J-I, Araki K, Yamato E et al. (1990) Establishment of a pancreatic $\beta$ cell line that retains glucose-inducible insulin secretion: special reference to expression of glucose transporter isoforms. Endocrinology 127: 126-132

8. Nishi M, Chan SJ, Nagamatsu S, Bell GI, Steiner DF (1989) Conservation of the sequence of islet amyloid polypeptide in five mammals is consistent with its putative role as an islet hormone. Proc Natl Acad Sci U S A 86: 5738-5742

9. Wakasugi S, Maeda S, Shimada K, Nakashima H, Migita S (1985) Structural comparisons between mouse and human prealbumin. J Biochem 98: 1707-1714

10. Walker JE, Arizmendi JM, Dupuis A et al. (1992) Sequences of 20 subunits of NADH:ubiquinone oxidoreductase from bovine heart mitochondria. Application of a novel strategy for sequencing proteins using the polymerase chain reaction. J Mol Biol 226: 1051-1072

11. von Lindern M, Fornerod M, van Baal S, Jaegle M, de Wit T, Buijs A, Grosveld G (1992) The translocation (6;9), associated with a specific subtype of acute myeloid leukemia, results in the fusion of two genes, dek and can, and the expression of a chimeric, leukemia-specific dek-can mRNA. Mol Cell Biol 12: 1687-1697

12. Weeda G, Ma L, van HR, Bootsma D, van dEA, Hoeijmakers JH (1991) Characterization of the mouse homolog of the XPBC/ERCC-3 gene implicated in xeroderma pigmentosum and Cockayne's syndrome. Carcinogenesis 12: 2361-2368
13. Michaud EJ, van Vugt MJ, Bultman SJ, Sweet HO, Davisson MT, Woychik RP (1994) Differential expression of a new dominant agouti allele (Aiapy) is correlated with methylation state and is influenced by parental lineage. Genes Dev 8: 1463-1472

14. Hubank M, Schatz DG (1994) Identifying differences in mRNA expression by representational difference analysis of cDNA. Nucleic Acids Res 22: 5640-5648

15. Novials A, Sarri Y, Casamitjana R, Rivera F, Gomis R (1993) Regulation of islet amyloid polypeptide in human pancreatic islets. Diabetes 42: 1514-1519

16. Jacobsson B (1989) In situ localization of transthyretinmRNA in the adult human liver, choroid plexus and pancreatic islets and in endocrine tumours of the pancreas and gut. Histochemistry 91: 299-304

17. Jacobsson B, Carlstrom A, Platz A, Collins VP (1990) Transthyretin messenger ribonucleic acid expression in the pancreas and in endocrine tumors of the pancreas and gut. J Clin Endocrinol Metab 71: 875-880

18. Itoh N, Hanafusa T, Miyagawa J (1992) Transthyretin (prealbumin) in the pancreas and sera of newly diagnosed type I (insulin-dependent) diabetic patients. J Clin Endocrinol Metab 74: 1372-1377

19. von Lindern M, Poustka A, Lerach H, Grosveld G (1990) The (6;9) chromosome translocation with a specific subtype of acute nonlymphocytic leukemia, leads to aberrant transcription of a target on 9q34. Mol Cell Biol 10: 4016-4026

20. Adams MD, Kelley JM, Gocayne JD et al. (1991) Complementary DNA sequencing: expressed sequence tags and human genome project. Science 252: 1651-1656

21. Adams MD, Dubnick M, Kerlavage AR et al. (1992) Sequence identification of 2,375 human brain genes. Nature 355: 632-634

22. Okubo K, Hori N, Matoba R et al. (1992) Large scale cDNA sequencing for analysis of quantitative and qualitative aspects of gene expression. Nat Genet 2: 173-179

23. Khan AS, Wilcox AS, Polymeropoulos MH et al. (1992) Single pass sequencing and physical and genetic mapping of human brain cDNAs. Nat Genet 2: 180-185

24. Adams MD, Kerlavage AR, Fields C, Venter JC (1993) 3,400 new expressed sequence tags identify diversity of transcripts in human brain. Nat Genet 4: 256-267

25. Höög C (1991) Isolation of a large number of novel mammalian genes by a diffential cDNA library screening startegy. Nucleic Acids Res 19: 6123-6127

26. Takeda J, Yano H, Eng S, Zeng Y, Bell GI (1993) A molecular inventory of human pancreatic islets: sequence analysis of 1000 cDNA clones. Hum Mol Genet 2: 1793-1798

27. Tanaka M, Katashima R, Murakami D et al. (1995) Molecular cloning of a group of mouse pancreatic islet beta-cellrelated genes by random cDNA sequenceing. Diabetologia 38: 381-386 